\title{
Current State of the Art in Distributed Autonomous Mobile Robotics
}

\author{
Lynne E. Parker \\ Center for Engineering Science Advanced Research \\ Computer Science and Mathematics Division \\ Oak Ridge National Laboratory, Oak Ridge TN 37831-6355, USA \\ email: ParkerLE@ornl.gov
}

Keywords: Distributed robotics, survey, cooperative robotics, multi-robot systems

\begin{abstract}
As research progresses in distributed robotic systems, more and more aspects of multi-robot systems are being explored. This article surveys the current state of the art in distributed mobile robot systems. Our focus is principally on research that has been demonstrated in physical robot implementations. We have identified eight primary research topics within multi-robot systems - biological inspirations, communication, architectures, localization/mapping/exploration, object transport and manipulation, motion coordination, reconfigurable robots, and learning - and discuss the current state of research in these areas. As we describe each research area, we identify some key open issues in multi-robot team research. We conclude by identifying several additional open research issues in distributed mobile robotic systems.
\end{abstract}

\section{Introduction}

The field of distributed robotics has its origins in the late-1980's, when several researchers began investigating issues in multiple mobile robot systems. Prior to this time, research had concentrated on either single robot systems or distributed problem-solving systems that did not involve robotic components. The topics of particular interest in this early distributed robotics work include:

- Cellular (or reconfigurable) robot systems, such as the work by Fukuda, et al. [20] on the Cellular Robotic System (CEBOT) and the work on cyclic swarms by Beni [8],

- Multi-robot motion planning, such as the work by Premvuti and Yuta [38] on traffic control and the work on movement in formations by Arai, et $a l$. [2] and Wang [48], and

- Architectures for multi-robot cooperation, such as the work on ACTRESS by Asama, et al. [4].

Since this early research in distributed mobile robotics, the field has grown dramatically, with a much wider variety of topics being addressed. This paper 
examines the current state of the art in autonomous multiple mobile robotic systems. The field of cooperative autonomous mobile robotics is still so new that no topic area within this domain can be considered mature. Some areas have been explored more extensively, however, and the community is beginning to understand how to develop and control certain aspects of multi-robot teams. Thus, rather than summarize the research into a taxonomy of cooperative systems (see Dudek [18] and Cao [12] for previous related summaries), we instead organize this research by the principal topic areas that have generated significant levels of study, to the extent possible in a limited space. As we present the review, we identify key open research issues within each topic area. We conclude by suggesting additional research issues that have not yet been extensively studied, but appear to be of growing interest and need in distributed autonomous multi-robot systems.

\section{Biological Inspirations}

Nearly all of the work in cooperative mobile robotics began after the introduction of the new robotics paradigm of behavior-based control [10,3]. This behavior-based paradigm has had a strong influence in much of the cooperative mobile robotics research. Because the behavior-based paradigm for mobile robotics is rooted in biological inspirations, many cooperative robotics researchers have also found it instructive to examine the social characteristics of insects and animals, and to apply these findings to the design of multi-robot systems ${ }^{1}$.

The most common application of this knowledge is in the use of the simple local control rules of various biological societies - particularly ants, bees, and birds - to the development of similar behaviors in cooperative robot systems. Work in this vein has demonstrated the ability for multi-robot teams to flock, disperse, aggregate, forage, and follow trails (e.g., [30,15,17]). The application of the dynamics of ecosystems has also been applied to the development of multi-robot teams that demonstrate emergent cooperation as a result of acting on selfish interests [32]. To some extent, cooperation in higher animals, such as wolf packs, has generated advances in cooperative control. Significant study in predator-prey systems has occurred, although primarily in simulation $[7,21]$. Competition in multi-robot systems, such as found in higher animals including humans, is beginning to be studied in domains such as multi-robot soccer [46,29].

These areas of biological inspiration and their applicability to multi-robot teams seem to be fairly well understood. More recently identified, less well understood, biological topics of relevance include the use of imitation in higher

\footnotetext{
${ }^{1}$ For a more detailed analysis of various types of biological systems - what Tinbergen called differentiating (e.g., ants) and integrative (e.g., birds) - and their relationship to cooperative robotics work (i.e., "swarm" vs. "intentional"), see $[35]$.
} 
animals to learn new behaviors, and the physical interconnectivity demonstrated by insects such as ants to enable collective navigation over challenging terrains.

\section{Communication}

The issue of communication in multi-robot teams has been extensively studied since the inception of distributed robotics research. Distinctions between implicit and explicit communication are usually made, in which implicit communication occurs as a side-effect of other actions, or "through the world", whereas explicit communication is an specific act designed solely to convey information to other robots on the team. Several researchers have studied the effect of communication on the performance of multi-robot teams in a variety of tasks, and have concluded that communication provides certain benefit for particular types of tasks $[27,6]$. Additionally, these researchers have found that, in many cases, communication of even a small amount of information can lead to great benefit [6].

More recent work in multi-robot communication has focused on representations of languages and the grounding of these representations in the physical world $[22,23]$. Additionally, work has extended to achieving fault tolerance in multi-robot communication, such as setting up and maintaining distributed communications networks [51] and ensuring reliability in multi-robot communications [34]. While progress is being made in these more recent issues of communication, much work remains to enable multi-robot teams to operate reliably amidst faulty communication environments.

\section{Architectures, Task Planning, and Control}

A great deal of research in distributed robotics has focused on the development of architectures, task planning capabilities, and control. This research area addresses the issues of action selection, delegation of authority and control, the communication structure, heterogeneity versus homogeneity of robots, achieving coherence amidst local actions, resolution of conflicts, and other related issues. Each architecture that has been developed for multirobot teams tends to focus on providing a specific type of capability to the distributed robot team. Capabilities that have been of particular emphasis include task planning [1], fault tolerance [36], swarm control [31], human design of mission plans [26], and so forth.

A general research question in this vein is whether specialized architectures for each type of robot team and/or application domain are needed, or whether a more general architecture can be developed that can be easily tailored to fit a wider range of multi-robot systems. Relatively little of the previous work has been aimed at unifying these architectures. Perhaps an allencompassing architecture would be too unwieldy to implement in practical 
applications. It remains to be seen if a single general architecture for multirobot teams can be developed that is applicable to a much wider variety of domains than is currently possible with existing architectures.

\section{Localization, Mapping, and Exploration}

An extensive amount of research has been carried out in the area of localization, mapping, and exploration for single autonomous robots. Only fairly recently has much of this work been applied to multi-robot teams. Almost all of the work has been aimed at 2D environments. Additionally, nearly all of this research takes an existing algorithm developed for single robot mapping, localization, or exploration, and extends it to multiple robots, as opposed to developing a new algorithm that is fundamentally distributed. One exception is some of the work in multi-robot localization, which takes advantage of multiple robots to improve positioning accuracy beyond what is possible with single robots $[42,19]$.

As is the case with single robot approaches to localization, mapping, and exploration, research into the multi-robot version can be described using the familiar categories based on the use of landmarks [14], scan-matching [11], and/or graphs $[40,39]$, and which use either range sensors (such as sonar or laser) or vision sensors. While the single robot version of this problem is fairly well understood, much remains to be studied in the multi-robot version. For example, one question is the effectiveness of multi-robot teams over single-robot versions, and to what extent adding additional robots brings diminishing returns. This issue has begun to be studied (see [39]), but much much remains to be determined for the variety of approaches available for localization, mapping, and exploration.

\section{Object Transport and Manipulation}

Enabling multiple robots to cooperatively carry, push, or manipulate common objects has been a long-standing, yet difficult, goal of multi-robot systems. Many research projects have dealt with this topic area; fewer of these projects have been demonstrated on physical robot systems. This research area has a number of practical applications that make it of particular interest for study.

Numerous variations on this task area have been studied, including constrained and unconstrained motions, two-robot teams versus "swarm"-type teams, compliant versus non-compliant grasping mechanisms, cluttered versus uncluttered environments, global system models versus distributed models, and so forth. Perhaps the most demonstrated task involving cooperative transport is the pushing of objects by multi-robot teams [43,45]. This task seems inherently easier than the carry task, in which multiple robots must grip common objects and navigate to a destination in a coordinated fashion 
$[49,24]$. A novel form of multi-robot transportation that has been demonstrated is the use of ropes wrapped around objects to move them along desired trajectories [16].

Nearly all of the previous work in this area work involves robots moving across a flat surface. A challenging open issue in this area is cooperative transport over uneven outdoor terrains.

\section{Motion Coordination}

A popular topic of study in multi-robot teams is that of motion coordination. Research themes in this domain that have been particularly well studied include multi-robot path planning [52], traffic control [38], formation generation [2], and formation keeping [5,48]. Most of these issues are now fairly well understood, although demonstration of these techniques in physical multi-robot teams (rather than in simulation) has been limited. More recent issues studied within the motion coordination context are target tracking [37], target search [25], and multi-robot docking [33] behaviors.

Nearly all of the previous work has been aimed at 2D domains, although some work has been aimed at 3D environments [52]. One of the most limiting characteristics of much of the existing path planning work is the computational complexity of the approaches. Perhaps as computing processor speed increases, the computational time will take care of itself. In the meantime, this characteristic is a limiting factor to the applicability of much of the path planning research in dynamic, real-time robot teams.

\section{Reconfigurable Robotics}

Even though some of the earliest research in distributed robotics focused on concepts for reconfigurable distributed systems [20,8], relatively little work has proceeded in this area until the last few years. More recent work has resulted in a number of actual physical robot systems that are able to reconfigure. The motivation of this work is to achieve function from shape, allowing individual modules, or robots, to connect and re-connect in various ways to generate a desired shape to serve a needed function. These systems have the theoretical capability of showing great robustness, versatility, and even self-repair.

Most of the work in this area involves identical modules with interconnection mechanisms that allow either manual or automatic reconfiguration. These systems have been demonstrated to form into various navigation configurations, including a rolling track motion [53], an earthworm or snake motion [53,13], and a spider or hexapod motion [53,13]. Some systems employ a cube-type arrangement, with modules able to connect in various ways to form matrices or lattices for specific functions $[9,54,44,47]$. 
Research in this area is still very young, and most of the systems developed are not yet able to perform beyond simple laboratory experiments. While the potential of large numbers of robot modules has, to some extent, been demonstrated in simulation, it is still uncommon to have implementations involving more than a dozen or so physical modules. The practical application of these systems is yet to be demonstrated, although progress is being made in that direction. Clearly, this is a rich area for continuing advances in multirobot systems.

\section{Learning}

Many multi-robot researchers believe that an approach with more potential for the development of cooperative control mechanisms is autonomous learning. While a considerable amount of work has been done in this area for multi-agent learning [50], somewhat less work has been accomplished to date in multi-robot learning. The types of applications that are typically studied for this area of multi-robot learning vary considerably in their characteristics. Some of the applications include predator/prey [7,21], box pushing [28], foraging [31], multi-robot soccer $[46,29,41]$, and cooperative target observation [37].

Particularly challenging domains for multi-robot learning are those tasks that are inherently cooperative - that is, tasks in which the utility of the action of one robot is dependent upon the current actions of the other team members. Inherently cooperative tasks cannot be decomposed into independent subtasks to be solved by a distributed robot team. Instead, the success of the team throughout its execution is measured by the combined actions of the robot team, rather than the individual robot actions. This type of task is a particular challenge in multi-robot learning, due to the difficulty of assigning credit for the individual actions of the robot team members. Multi-robot learning in general, and inherently cooperative task learning in particular, are areas in which significant research for multi-robot systems remains.

\section{Additional Open Issues in Distributed Autonomous Mobile Robotics}

It is clear that since the inception of the field of distributed autonomous mobile robotics less than two decades ago, significant progress has been made on a number of important issues. The field has a good understanding of the biological parallels that can be drawn, the use of communication in multi-robot teams, and the design of architectures for multi-robot control. Considerable progress has been made in multi-robot localization/mapping/exploration, cooperative object transport, and motion coordination. Recent progress is beginning to advance the areas of reconfigurable robotics and multi-robot 
learning. Of course, all of these areas have not yet been fully studied; we have identified key open research challenges for these areas in the previous sections.

Several other research challenges still remain, including:

- How do we identify and quantify the fundamental advantages and characteristics of multi-robot systems?

- How do we easily enable humans to control multi-robot teams?

- Can we scale up to demonstrations involving more than a dozen or so robots?

- Is passive action recognition in multi-robot teams possible?

- How can we enable physical multi-robot systems to work under hard real-time constraints?

- How does the complexity of the task and of the environment affect the design of multi-robot systems?

These and other issues in multi-robot cooperation should keep the research community busy for many years to come.

\section{Acknowledgments}

This work is sponsored by the Engineering Research Program of the Office of Basic Energy Sciences, U. S. Department of Energy. Accordingly, the U.S. Government retains a nonexclusive, royalty-free license to publish or reproduce the published form of this contribution, or allow others to do so, for U. S. Government purposes. Oak Ridge National Laboratory is managed by UT-Battelle, LLC for the U.S. Dept. of Energy under contract DE-AC05-00OR22725.

\section{References}

1. R. Alami, S. Fleury, M. Herrb, F. Ingrand, and F. Robert. Multi-robot cooperation in the Martha project. IEEE Robotics and Automation Magazine, 1997.

2. T. Arai, H. Ogata, and T. Suzuki. Collision avoidance among multiple robots using virtual impedance. In Proceedings of the Intelligent Robots and Systems (IROS), pages 479-485, 1989.

3. Ronald C. Arkin. Integrating behavioral, perceptual, and world knowledge in reactive navigation. Robotics and Autonomous Systems, 6:105-122, 1990.

4. Hajime Asama, Akihiro Matsumoto, and Yoshiki Ishida. Design of an autonomous and distributed robot system: ACTRESS. In Proceedings of IEEERSJ International Workshop on Intelligent Robots and Systems, pages 283-290, Tsukuba, Japan, 1989.

5. T. Balch and R. Arkin. Behavior-based formation control for multi-robot teams. IEEE Transactions on Robotics and Automation, December 1998.

6. Tucker Balch and Ronald C. Arkin. Communication in reactive multiagent robotic systems. Autonomous Robots, 1(1):1-25, 1994. 
7. M. Benda, V. Jagannathan, and R. Dodhiawalla. On optimal cooperation of knowledge sources. Technical Report BCS-G2010-28, Boeing AI Center, August 1985.

8. Gerardo Beni. The concept of cellular robot. In Proceedings of Third IEEE Symposium on Intelligent Control, pages 57-61, Arlington, Virginia, 1988.

9. H. Bojinov, A. Casal, and T. Hogg. Emergent structures in moduluar selfreconfigurable robots. In Proceedings of the IEEE International Conference on Robotics and Automation, pages 1734-1741, 2000.

10. Rodney A. Brooks. A robust layered control system for a mobile robot. IEEE Journal of Robotics and Automation, RA-2(1):14-23, March 1986.

11. W. Burgard, M. Moors, D. Fox, R. Simmons, and S. Thrun. Collaborative multi-robot exploration. In Proceedings of the IEEE International Conference on Robotics and Automation, pages 476-481, 2000.

12. Y. Uny Cao, Alex Fukunaga, Andrew Kahng, and Frank Meng. Cooperative mobile robotics: Antecedents and directions. In Proceedings of 1995 IEEE/RSJ International Conference on Intelligent Robots and Systems (IROS '95), pages 226-234, 1995.

13. A. Castano, R. Chokkalingam, and P. Will. Autonomous and self-sufficient conro modules for reconfigurable robots. In Proceedings of the Fifth International Symposium on Distributed Autonomous Robotic Systems (DARS 2000 this volume), 2000.

14. G. Dedeoglu and G. Sukhatme. Landmark-based matching algorithm for cooperative mapping by autonomous robots. In Proceedings of the Fifth International Symposium on Distributed Autonomous Robotic Systems (DARS 2000 this volume), 2000.

15. J. Deneubourg, S. Goss, G. Sandini, F. Ferrari, and P. Dario. Self-organizing collection and transport of objects in unpredictable environments. In JapanU.S.A. Symposium on Flexible Automation, pages 1093-1098, 1990.

16. B. Donald, L. Gariepy, and D. Rus. Distributed manipulation of multiple objects using ropes. In Proceedings of IEEE International Conference on Robotics and Automation, pages 450-457, 2000.

17. Alexis Drogoul and Jacques Ferber. From Tom Thumb to the Dockers: Some experiments with foraging robots. In Proceedings of the Second International Conference on Simulation of Adaptive Behavior, pages 451-459, 1992.

18. Gregory Dudek et al. A taxonomy for swarm robots. In Proceedings of 1993 IEEE International Conference on Intelligent Robots and Systems (IROS '93), pages 441-447, 1993.

19. D. Fox, W. Burgard, H. Kruppa, and S. Thrun. Collaborative multi-robot exploration. Autonomous Robots, 8(3), 2000.

20. T. Fukuda and S. Nakagawa. A dynamically reconfigurable robotic system (concept of a system and optimal configurations). In Proceedings of IECON, pages 588-595, 1987.

21. Thomas Haynes and Sandip Sen. Evolving behavioral strategies in predators and prey. In Gerard Weiss and Sandip Sen, editors, Adaptation and Learning in Multi-Agent Systems, pages 113-126. Springer, 1986.

22. L. Hugues. Collective grounded representations for robots. In Proceedings of Fifth International Conference on Distributed Autonomous Robotic Systems (DARS 2000 - this volume), 2000.

23. David Jung and Alexander Zelinsky. Grounded symbolic communication between heterogeneous cooperating robots. Autonomous Robots, 8(3), July 2000. 
24. O. Khatib, K. Yokoi, K. Chang, D. Ruspini, R. Holmberg, and A. Casal. Vehicle/arm coordination and mobile manipulator decentralized cooperation. In IEEE/RSJ International Conference on Intelligent Robots and Systems, pages 546-553, 1996.

25. S. M. LaValle, D. Lin, L. J. Guibas, J-C. Latombe, and R. Motwani. Finding an unpredictable target in a workspace with obstacles. In submitted to 1997 International Conference on Robots and Automation, 1997.

26. D. MacKenzie, R. Arkin, and J. Cameron. Multiagent mission specification and execution. Autonomous Robots, 4(1):29-52, 1997.

27. Bruce MacLennan. Synthetic ethology: An approach to the study of communication. In Proceedings of the 2nd interdisciplinary workshop on synthesis and simulation of living systems, pages 631-658, 1991.

28. S. Mahadevan and J. Connell. Automatic programming of behavior-based robots using reinforcement learning. In Proceedings of AAAI-91, pages 8-14, 1991.

29. S. Marsella, J. Adibi, Y. Al-Onaizan, G. Kaminka, I. Muslea, and M. Tambe. On being a teammate: Experiences acquired in the design of RoboCup teams. In O. Etzioni, J. Muller, and J. Bradshaw, editors, Proceedings of the Third Annual Conference on Autonomous Agents, pages 221-227, 1999.

30. Maja Mataric. Designing emergent behaviors: From local interactions to collective intelligence. In J. Meyer, H. Roitblat, and S. Wilson, editors, Proc. of the Second Int'l Conf. on Simulation of Adaptive Behavior, pages 432-441. MIT Press, 1992.

31. Maja Mataric. Interaction and Intelligent Behavior. PhD thesis, Massachusetts Institute of Technology, 1994.

32. David McFarland. Towards robot cooperation. In D. Cliff, P. Husbands, J.-A. Meyer, and S. Wilson, editors, Proceedings of the Third International Conference on Simulation of Adaptive Behavior, pages 440-444. MIT Press, 1994.

33. B. Minten, R. Murphy, J. Hyams, and M. Micire. A communication-free behavior for docking mobile robots. In Proceedings of Fifth International Symposium on Distributed Autonomous Robotic Systems (DARS 2000 - this volume), 2000.

34. P. Molnar and J. Starke. Communication fault tolerance in distributed robotic systems. In Proceedings of Fifth International Symposium on Distributed Autonomous Robotic Systems (DARS 2000 - this volume), 2000.

35. L. E. Parker. Adaptive action selection for cooperative agent teams. In JeanArcady Meyer, Herbert Roitblat, and Stewart Wilson, editors, Proceedings of the Second International Conference on Simulation of Adaptive Behavior, pages 442-450. MIT Press, 1992.

36. L. E. Parker. ALLIANCE: An architecture for fault-tolerant multi-robot cooperation. IEEE Transactions on Robotics and Automation, 14(2):220-240, 1998.

37. L. E. Parker. Multi-robot learning in a cooperative observation task. In Proceedings of Fifth International Symposium on Distributed Autonomous Robotic Systems (DARS 2000 - this volume), 2000.

38. Suparerk Premvuti and Shin'ichi Yuta. Consideration on the cooperation of multiple autonomous mobile robots. In Proceedings of the IEEE International Workshop of Intelligent Robots and Systems, pages 59-63, Tsuchiura, Japan, 1990 . 
39. N. S. V. Rao. Terrain model acquisition by mobile robot teams and nconnectivity. In Proceedings of the Fifth International Symposium on Distributed Autonomous Robotic Systems (DARS 2000 - this volume), 2000.

40. I. Rekleitis, G. Dudek, and E. Milios. Graph-based exploration using multiple robots. In Proceedings of the Fifth International Symposium on Distributed Autonomous Robotic Systems (DARS 2000 - this volume), 2000.

41. P. RIley and M. Veloso. On behavior classification in adversarial environmentts. In Proceedings of Fifth International Symposium on Distributed Autonomous Robotic Systems (DARS 2000 - this volume), 2000.

42. S. Roumeliotis and G. Bekey. Distributed multi-robot localization. In Proceedings of the Fifth International Symposium on Distributed Autonomous Robotic Systems (DARS 2000 - this volume), 2000.

43. D. Rus, B. Donald, and J. Jennings. Moving furniture with teams of autonomous robots. In Proceedings of IEEE/RSJ International Conference on Intelligent Robots and Systems, pages 235-242, 1995.

44. D. Rus and M. Vona. A physical implementation of the self-reconfiguring crystalline robot. In Proceedings of the IEEE International Conference on Robotics and Automation, pages 1726-1733, 2000.

45. Daniel Stilwell and John Bay. Toward the development of a material transport system using swarms of ant-like robots. In Proceedings of IEEE International Conference on Robotics and Automation, pages 766-771, 1993.

46. P. Stone and M. Veloso. A layered approach to learning client behaviors in the robocup soccer server. Applied Artificial Intelligence, 12:165-188, 1998.

47. C. Unsal and P. K. Khosla. Mechatronic design of a modular self-reconfiguring robotic system. In Proceedings of the IEEE International Conference on Robotics and Automation, pages 1742-1747, 2000.

48. P. K. C. Wang. Navigation strategies for multiple autonomous mobile robots. In Proceedings of the IEEE/RSJ International Conference on Intelligent Robots and Systems (IROS), pages 486-493, 1989.

49. Z. Wang, Y. Kimura, T. Takahashi, and E. Nakano. A control method of a multiple non-holonomic robot system for cooperative object transportation. In Proceedings of Fifth International Symposium on Distributed Autonomous Robotic Systems (DARS 2000 - this volume), 2000.

50. Gerhard Weiss and Sandip Sen, editors. Adaption and Learning in Multi-Agent Systems. Springer, 1996.

51. A. Winfield. Distributed sensing and data collection via broken ad hoc wireless connected networks of mobile robots. In Proceedings of Fifth International Symposium on Distributed Autonomous Robotic Systems (DARS 2000 - this volume), 2000.

52. A. Yamashita, M. Fukuchi, J. Ota, T. Arai, and H. Asama. Motion planning for cooperative transportation of a large object by multiple mobile robots in a 3d environment. In Proceedings of IEEE International Conference on Robotics and Automation, pages 3144-3151, 2000.

53. M. Yim, D. G. Duff, and K. D. Roufas. Polybot: a modular reconfigurable robot. In Proceedings of the IEEE International Conference on Robotics and Automation, pages 514-520, 2000.

54. E. Yoshida, S. Murata, S. Kokaji, and K. Tomita dn H. Kurokawa. Micro self-reconfigurable robotic system using shape memory alloy. In Proceedings of the Fifth International Symposium on Distributed Autonomous Robotic Systems (DARS 2000 - this volume), 2000. 